\title{
Towards a Model of a Scalable Middleware Architecture Based on Cloud Computing, Application Reliable Integration and E-Learning Platforms in Academic Information Systems
}

\author{
Abdellah Lalaoui Hassani, Khalifa Mansouri \\ Laboratory: Signals, Distributed Systems and Artificial Intelligence (SSDIA) \\ ENSET Mohammedia, University Hassan II OF \\ Casablanca, Morocco \\ lalaoui_abdellah@yahoo.fr; khmansouri@hotmail.com
}

\begin{abstract}
Throughout the present article, we will examine, on the one hand, systems integration approaches of eLearning applications in university's information systems. On the other hand, we will establish a study about the integration of applications between the internal information systems and the Cloud. Then between the applications on the private cloud and the public cloud.
\end{abstract}

Keywords-E-Learning; Higher Education; University's Information System; Middleware Architecture; Integration; EAI; SOA; ESB; Cloud computing.

\section{Introduction}

For more than a decade, the world of information systems has gradually invaded the Moroccan higher education sector. Universities, engineering schools, public and private higher education schools, are making great strides for an optimal use of information and communication technologies (ICT).

Several institutions have urbanized their information systems by either integrating management softwares such as Enterprise Resource Planning (ERP) [16] or locally developing systems, that are basically dedicated to manage:

- School affairs: notes, absences, timetables, agendas, etc;

- Internal and external communication: messaging, staff's information;

- Educational means/tools: digital textbook, document storage, collaborative tools, blogs, forum etc.

Those information systems are most or time developed to be accessible from any device (desktop, laptop, tablet, smart-phone) connected to the Internet. There are several software tools of managing school governance and school life management, for example :

The APOGEE system as a standard, is a paying ERP and the most used by universities. It meet, practically the majority of their own needs [6];

The GESFO system in particular, is a ERP developed locally by the IT Department of ISGA (Higher Institute of Engineering and Business) [10].

Figure 1 shows the general layout of the IS architecture within a Higher Education institution : 


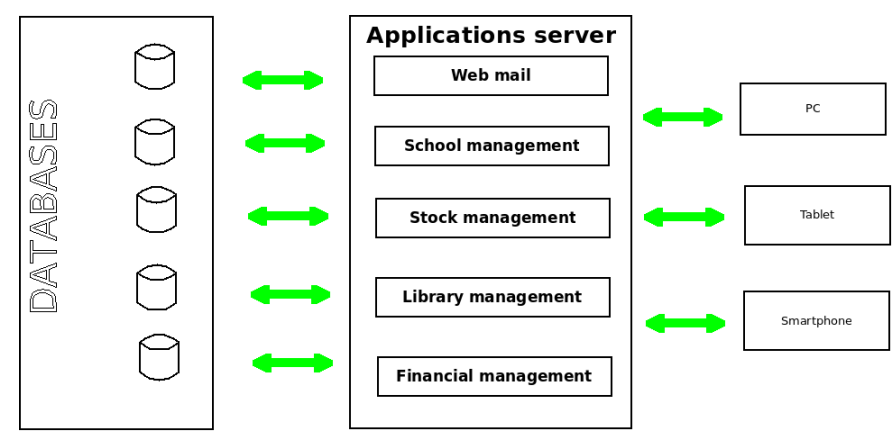

Figure. 1 IS architecture within an institution

ICT has greatly infested the world of pedagogy in Morocco [6], so to offer a large-scale interaction participation and an efficient control of its different users. for example, distance education, trough the use of e-learning

systems [7]. Those systems represent, first of all, a new teaching method that saves a lot of time, shares knowledge, builds skills and know-how. Secondly, they improve learning services for learners [8].

In addition, the Moroccan Ministry of Higher Education, Training of Managers and Scientific Research has shown a strong desire to successfully integrate e-Learning in education. Indeed, the managers are aware that this integration will surely improve the quality of teaching and learning. For this purpose, it is necessary that some Moroccan institutions aligns on international standards in terms of integration and use of e-Learning platform officially in their educational system. However, it is a large scale project, whose encounters so many difficulties and obstacles.

This article presents two studies:

The first one is about applications integration's field in a university's information system and its relationships with an e-learning platform.

The second one is related to the integration of applications between the internal information system and the Cloud (scenario IS Campus to Cloud and Cloud to IS Campus) then between applications on the Cloud (scenario from private cloud to public cloud).

After an introduction, which basically aimed at a contextualization of the concept of integration, we will present in a first section an overview about the different platforms of application integration, starting from the point-to-point architecture to the ESB (Enterprise Service Bus) architecture [3], by passing through EAl architecture (Enterprise Application Integration) [1]. Then, we will identify highlights of the ESB architecture.

We shall present, in the second section of the article a modern and a service-oriented architecture (SOA) system's structure and integration infrastructure in between two internal information systems (IS2IS)[3] and that, by using an enterprise service bus (ESB). Its aim is to create more flexible solutions for integration and coupling of systems. The third section of the article displays a presentation of cloud computing and as-well-as its main deployment model. 
Thereafter, we will expose the advantages of e-learning based on the Cloud and its differences compared to e-learning based on a basic infrastructure. The last section presents a new model that is more relevant and beneficial for universities. This model combines the ESB integration solution and the Cloud computing.

\section{Context of Our Work and Problematic Proposal}

Today, we cannot imagine a software that can fit all the needs expressed by a company. An Information System (IS) is an assembly of independent applications, each of which is designed to meet a specific need. In order for the IT department to suit the requirements of the company, it tries to take the best customer relationship management (CRM)[16] solution, the best Enterprise Resource Planning Software (ERP) and the best Document Electronic Management solution (EDM)[9], so that in order to integrates them into its IS.

That is, as well, a particular problematic in the Higher Education sector. Following a study carried out on Moroccan institutions organized on several campuses, it was found that most of them gather several softwares (paying or open source) by means of heterogeneous solutions to adapt their University's Information Systems (UIS), with their specific learning management needs.

This configuration of the UIS causes a desynchronization of all the data between the applications. This will force users to duplicate information many times in different applications, and this could be a source of many input errors.

We have taken for this research, an institution of Private Higher Education in Morocco, composed of five training campuses and a general direction, spread over the national territory. In each campus, the information system is represented as follows:

Educ server: this server hosts an application named Educ that allows to manage all the business process of the school (students, absences, notes, teachers, etc.).

Educ DIRECT Server: This server hosts an application dedicated to tutors, in order to monitor the academic progression of the students. The data is replicated in real time between the two applications through a tool, which allows to automate the copying of the data between the Educ server and the EducDIRECT server in a unidirectional way. Each IS Campus works in an autonomous manner and there is no connection between them.

In 2015, the General Direction decided to integrate an e-Learning system as a complementary support to classical instruction called "face-to-face" (requiring the student's daily presence in the classroom). It chose the "Moodle" platform as an e-learning solution for training and distance learning. This platform operates independently of the other campus ISs.

It is hosted in the information system of the General Direction and regroup all students and teachers of the various campuses. With this solution, teachers can upload their courses in the platform and learners can take courses distantly.

Figure 2 shows the general layout of the overall architecture of the new IS within the institution studied by integrating the e-Learning platform. 


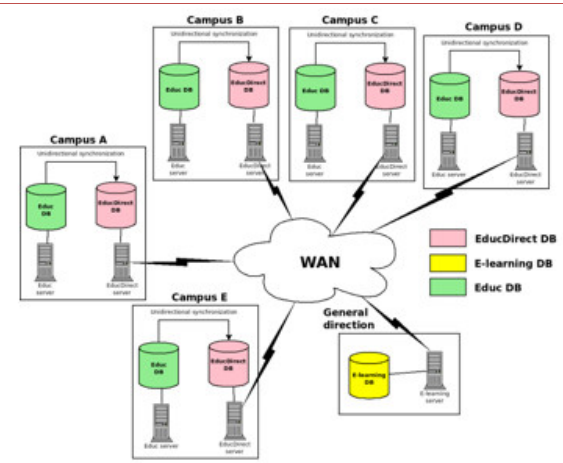

Figure. 2 Overall IS Architecture of the Institution Integrating the e-Learning Platform

This solution has raised a lot of problems at the business processes level of the campuses, particularly for the actors (students, teachers and operator of the studies department). These problems are:

Problem 1: Students and teachers have an access code for each application; therefore numerous access codes may be forgotten by their users.

Problem 2: The independence of this platform with ISs of the different campuses generates a delay to provide a synchronization of student and teacher accounts.

Problem 3: Operators are obliged to enter the same data twice (student information, notes, modules, user accounts, etc.) into each application, which requires a considerable time to feed the databases. Also, it may generate numerous errors from the data entered. The next figure presents an illustration of this problem:

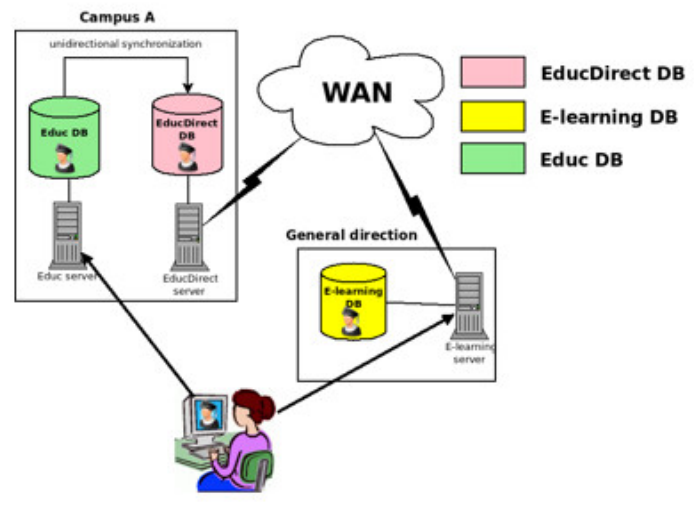

Figiure. 3 Illustration of problem 3, posed by the global architecture of IS.

Figure 3 shows that the operator connects to the Educ application in order to enter a new data of a student, then it must switch to the e-Learning system to re-enter the same information of that student.

\section{Proposal for a Technical Solution}

In order to provide a better user experience for learners, educators and managers and to avoid the incoherence in the data because of actions that are performed on a system but not on another, or to enter the same data in a variety of systems, it is necessary to ensure integration of information exchange between all these applications. 
Our approach is to propose an integration application platform. This platform allows heterogeneous applications to manage their exchanges while ensuring the confidentiality, integrity, speed of transfer and authenticity of the data. For instance, it allows an application, viewing and / or updating data from another application.

Figure 4 illustrates the different exchanges of data allowed between the e-Learning platform hosted in the information system of the general direction from any campus.

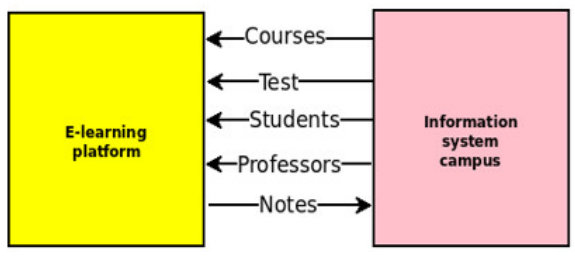

Figure. 4 Exchanges of data between the e-Learning platform and the IS campus

The integration of applications is an issue that was born with the evolution of the architectural and technological level of the software. According to Manouvrier, integration brings all the methods and tools that organize exchanges between the applications and the business processes of the company[1].

\subsection{Forms of integration}

Three forms of integration can be distinguished:

Integration of data;

Integration of specific treatments;

Integration of global applications.

Data integration is a form of integration dedicated to databases in order to facilitate their connectivity. This form allows the user to migrate, consolidate, combine all types of data from various sources: flat files, database, mail, etc.

Process integration is a form of integration that consist on integrating a new software entity within an application to provide an additional functionality, such as Application Programming Interface (API)[3].

Application integration consists on an approach that allows

Interconnecting distributed and heterogeneous applications developed independently in incompatible way, such as the case of two ERPs coming from two different editors.

Application Integration Platforms

Three approaches of architectures integration can be identified:

Point-to-point architecture;

EAI architecture;

ESB architecture. 
Abdellah Lalaoui Hassani, Khalifa Mansouri. Towards a Model of a Scalable Middleware Architecture Based on Cloud

\subsection{Point-to-point architecture}

It is an architecture based on a point-to-point integration approach. Each pair of applications is considered as separate brick, related to its own connectors and its own communication protocol (Figure 5).

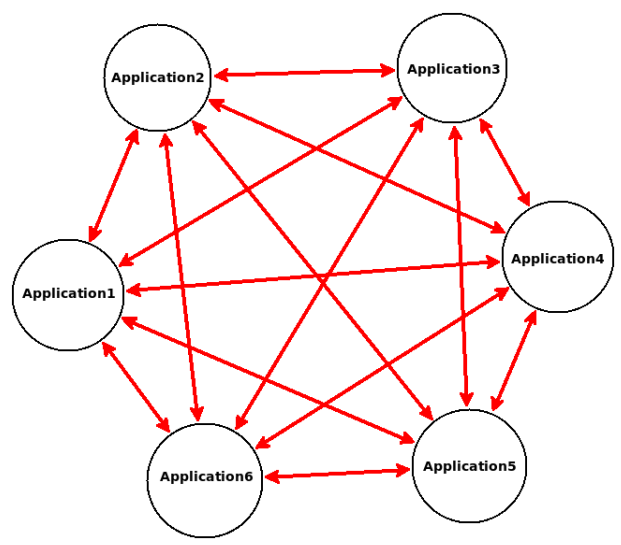

Figure. 5 Point-to-point integration architecture

This type of integration causes a confusion of proprietary and heterogeneous connections, which makes it intricate for application to be managed since the number of interfaces increases with the number of applications that need to be integrated into the information system.

\subsection{EAI Architecture}

This architecture is a centralized platform. Each application connects in a unique and independent way to the central system, without knowing a priori, the global topology of the information system (Figure 6).

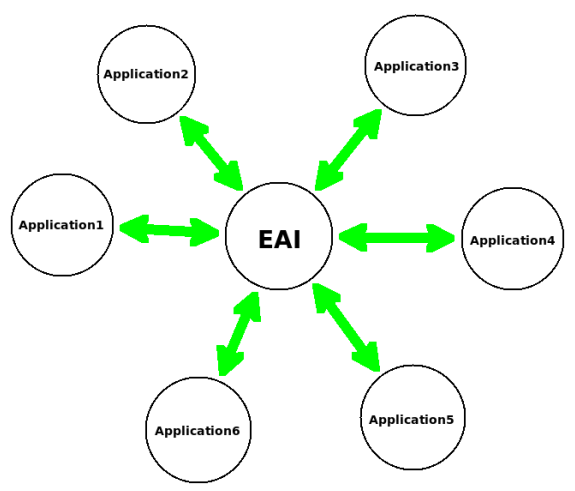

Figure. 6 EAl Integration Architecture

Despite its advantages, compared to the first architecture, in terms of robustness, reliability unification of connectors, and as in any centralized architecture, the main weakness of this architecture is the SPOF. (Single Point of Failure). Indeed any malfunction at the central system, leads to shutdown the entire platform. 


\subsection{Architecture ESB}

It is a fully distributed architecture, often used in the SOA context [11] and providing services such as data transmission, data transformation and increased interoperability through the systematic use of standards such as XML (Extensible Markup Language)[14] and WEB SERVICES [12], (Figure 7).

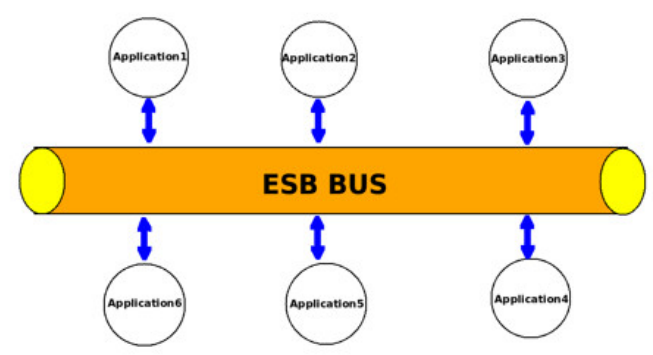

Figure. 7 ESB integration architecture

On the basis of this observation, and after comparing the different approaches mentioned above, we have opted to use the ESB integration architecture as an appropriate solution for our case study [11].

\section{Initial Implementation of the Esb Architecture in Our Case Study}

Figure 8 illustrates the implementation of the ESB Integration Architecture on a school campus.

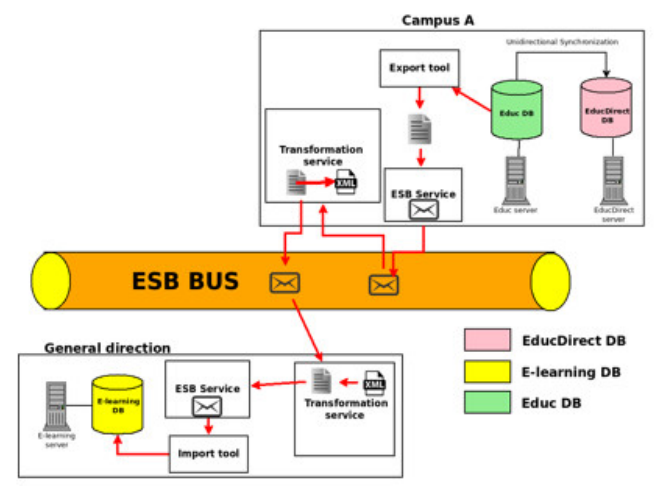

Figure. 8 Implementing the ESB Integration Architecture on Campus A

The data passing through the ESB bus is always represented by the file generated by the export tool. The transformation of the representation of this data in an XML form will allow to share this information on the bus.

The structure of the file generated by the export tool is understandable only by the import tool, located at the seat.

Data transformation takes place in the ESB bus. A transformation service is intercalated in the process of sending messages to the headquarters. This service is configured first, to recognize, the structure of the file generated by the export tool, second, to generate a better structured file.

The data transiting the ESB bus is not sent directly to the recipient, but will follow a circuit passing through different points of the ESB until finally reach the seat.. This circuit is defined directly in the message containing the data. 
Abdellah Lalaoui Hassani, Khalifa Mansouri. Towards a Model of a Scalable Middleware Architecture Based on Cloud Computing, Application Reliable Integration and E-Learning Platforms in Academic Information Systems . Transactions on Machine Learning and Artificial Intelligence, Vol 5 No 4 August (2017); p: 651-664

Returning to the problem number 3 that has been already explained in figure 3 and after the implementation of the ESB integration architecture, the campus A operator will enter data that concerns one student, only once on the Educ system. The data will pass through the ESB bus, as a generated file throughout the export tool. This file will be transformed into an XML form, through the transformation service. Once the XML file arrives to the general direction, The transformation service will convert this file into an understandable one by the import tool. Then this tool will send the file that contains the information of a student to the E-Learning database.

In the case of a technical problem that requires the restart of the bus or when a recipient (here, the general direction) is not reachable momentarily, after that the e-Learning server will shutdown, the nondelivered messages will be sent again to a backup system integrated in the bus [3].

\section{Improving the Proposed Solution}

The proposed architecture has considerably improved the business process of the institution studied, namely:

- $\quad$ Avoid to re-enter notes, student information, modules and matters;

- $\quad$ Avoid to recreate accounts of students and teachers;

- $\quad$ Avoid to give two different accounts to students and teachers;

- $\quad$ Adapting the mixed learning method with the information system such as course follow-up, inputting notes etc.

However, the data transmitted on the ESB bus is sometimes voluminous, which involve that the data transformation tool can take a long time in processing before transferring it to the recipient.

We have tried, through a new approach, to improve the transfer time of data sent by all applications.

To do this, we focused on two main points:

The nature of the data transferred;

The data transfer processing on the ESB bus.

\subsection{Nature of data transferred}

Applications that exist on campus and seat can generate data in XML format, Then we can add a coding tool in front of each application that will encode the data generated in XML to EXI and reciprocally EXI to XML.

EXI is a binary XML storage and data transfer format that has the same mechanism as the XML format, but is more compact, faster to exchange and process on the computer network [2].

\subsection{Data transfer processing on the ESB bus}

To reduce data transfer processing, we have moved the ESB bus directly in front of each encoding tool without having to connect with the export tool.

A JCA (Java Connector Architecture) adapter is used to connect the applications to the ESB.

This adapter allows sending information about, notes, students or teachers on the ESB bus. 
This information will be directly EXI elements of type note, student, teacher, module, subject as already seen. The same for the received data, they contain information in EXI format that will be interpreted and transmitted to the destination application (Figure 9).

Figure 9 illustrates the new version of the ESB architecture with improvements in the nature of the transferred data and the processing of data transfer on the ESB bus.

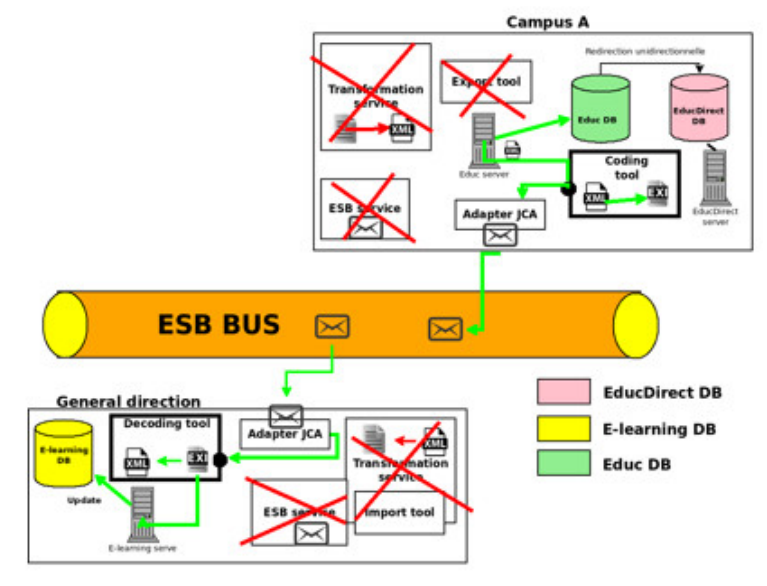

Figure. 9 The final version of the proposed ESB architecture

This change in the architecture of the institution's information system ensures the following advantages:

- $\quad$ The exchange of information between the various campuses and the seat is instantaneous and rational;

- Data is secured across the bus;

- $\quad$ Data transfer is fast through the bus;

- The information is represented in standardized form, and can be read and used from any application of the establishment;

- The information is entered once by the operators of the system.

Despite its technical advantages, this traditional online learning mode illustrates many disadvantages that can be presented in the following way:

- The development and maintenance of the systems is done by the educational institutions, which requires a permanent and competent IT team to ensure the evolution and high availability of the system;

- $\quad$ In order to ensure the well functioning of the e-Learning platform, the institutions must pay for software licenses, technical support for software and also verify that the software is installed on client devices;

- This architecture has no backup and restore policy, which can cause a big problem if the server crashes;

- $\quad$ Lack of an online collaboration and storage space for teachers and students which obliges them to back up and transfer files from one device to another. 
Abdellah Lalaoui Hassani, Khalifa Mansouri. Towards a Model of a Scalable Middleware Architecture Based on Cloud Computing, Application Reliable Integration and E-Learning Platforms in Academic Information Systems . Transactions on Machine Learning and Artificial Intelligence, Vol 5 No 4 August (2017); p: 651-664

\section{Proposal for a Technical Solution Based on the Cloud Computing}

Since the traditional e-learning solution is confronted with several problems related to resource optimization, storage, simultaneous data processing and also increasing costs, it becomes necessary to use an environment that meets both, scalable requirements and cost control. This environment is named the Cloud computing.

Our approach is to propose a modern, service-oriented infrastructure while maintaining the ESB middleware architecture that will ensure the integration of applications between firstly, two internal information systems (Scenario between a campus's internal IS and the e-Learning platform hosted in the general direction). Secondly, between the internal information system and the Cloud (Scenario between a campus's internal IS and the e-Learning platform hosted in the cloud) while ensuring high availability , Data security and IS to Cloud, Cloud to IS and Cloud to Cloud communication.

\subsection{The Cloud computing}

According to NIST [13], cloud computing is a model of a service platform that allows easy, on-demand access by the network to a shared set of computing resources (servers, storage, applications, and services). Cloud computing consists of four deployment models:

Private cloud: used by a single organization, it can be managed by the organization or by a third party. It can be placed in the organization's locals or outside.

Public cloud: open to the public. It is owned by an organization that sells Cloud services.

Community Cloud: shared by several organizations for the needs of a community that wish to pool resources (security, compliance, etc.)

Hybrid Cloud : composed of one or more models (public, private and community). These models are linked together by the same technology that allows portability of applications and data It is an excellent solution that allows to benefit from the advantages of private clouds and public clouds [15].

The National Institute for Standardization and Technology (NIST) divides cloud computing into two types of models:

Service models that refer to particular services accessible throughout the cloud computing platforms,

Deployment models that refer to the location and management of the Cloud infrastructure.

\subsection{E-Learning based on cloud computing}

With the increase of student's number, the rapid growth of educational content and the evolution of the IT infrastructure, educational institutions are facing the increasing costs and the decreasing budgets. Which requires to find an alternative for their e-Learning solutions.

Furthermore, current e-learning systems are not scalable and do not lead to efficient use of resources. In response to these barriers, institutions need to adopt service-oriented approaches.

The potential effectiveness of using cloud computing in higher education has been recognized by many international universities, such as the University of California, Washington State University School of 
Electrical Engineering and Computer Science, Higher Education Institutes of United Kingdom, the United States and others [12].

Institutions will be responsible of the process of education, content management and delivery while the service provider is responsible of the development, management and maintenance of the system. Establishments are charged according to the number of servers used that as well depends to the number of students as shown in figure 10.

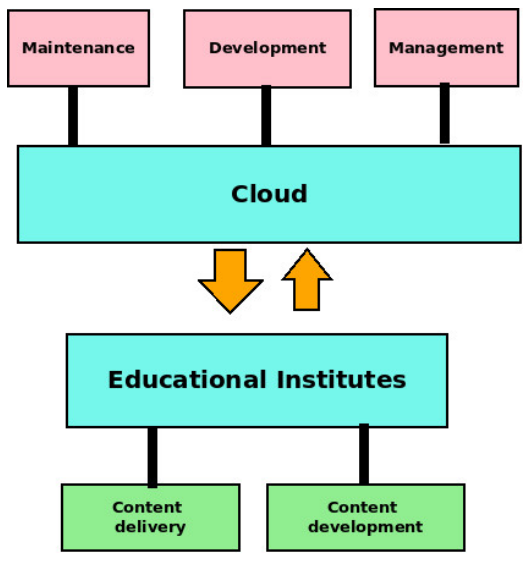

Figure. 10 Separation of roles in e-Learning based on Cloud

\subsection{Migrating the e-Learning platform to the Cloud}

The proposed solution must ensure the following [4]:

Continuity of information between internal IS and Cloud;

Continuity of information between applications on the Cloud;

Confidentiality of transferred data;

Confidentiality of existing data in IS Campus.

The features needed to integrate cloud-to-cloud and IS-to-cloud applications are the same. Both types rely on technical connectivity, data mapping, different exchange patterns, and so on.

The ESB exchange server and the e-Learning platform management module will need a maximum level of security, which requires to keep them in a private cloud.

The EducDirect application and the two modules related to teachers and students, in the e-Learning application platform, will need more computing power to respond to users requests, which requires to host them in a public cloud to a service provider .

To avoid losing IS Campus data, we have to set up an automatic backup system that records all IS Campus databases and stores them in a private cloud.

To meet our needs, the hybrid deployment model is the ideal solution in this case study [5]. However, this model points out a problem of communication between the private cloud and the public cloud related to security and performance.

In order to exchange fluently the flow of information between the two environments, it is essential to create links between the two clouds (private and public) by setting up a private network to promote 
Abdellah Lalaoui Hassani, Khalifa Mansouri. Towards a Model of a Scalable Middleware Architecture Based on Cloud Computing, Application Reliable Integration and E-Learning Platforms in Academic Information Systems . Transactions on Machine Learning and Artificial Intelligence, Vol 5 No 4 August (2017); p: 651-664

communication between the two systems without passing through the public Internet. This avoids all problems of failure, deceleration and safety.

Our proposed new architecture for e-learning based on cloud computing and the ESB bus is shown in Figure 11:

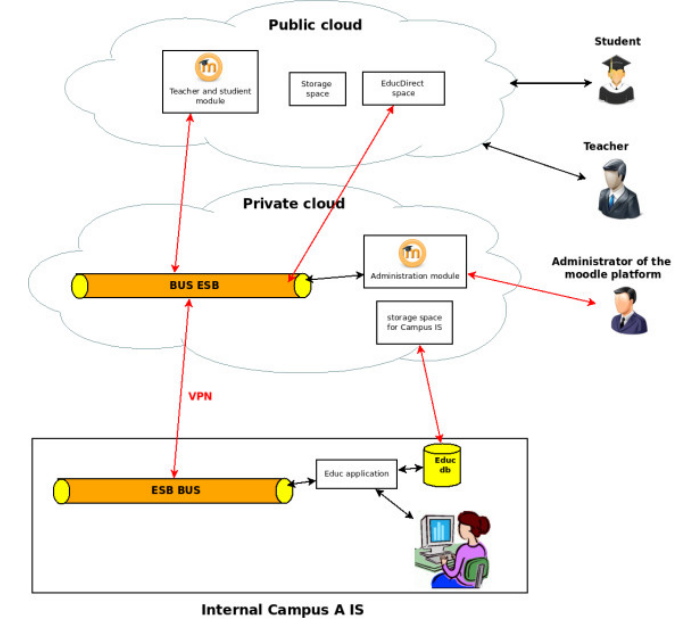

Figure. 11 E-learning model based on cloud computing and ESB

\section{E-Learning Cloud Based Architecture}

The advantages of the proposed architecture are:

\subsection{Powerful computing and storage capacity:}

Cloud computing is an infrastructure in which computing and storage power are managed by numerous distributed servers. Throughout cloud computing, users will connect securely to Internet.

The e-Learning architecture based on cloud puts data in a large number of distributed computers to provide high computing power and huge data storage space.

\subsection{High data security:}

In the Cloud computing model, the software and data are located on remote servers. Service providers manage unified data, allocate resources, balance load, deploy software, monitor security and perform reliable real-time monitoring, ensuring, as far as possible, user data security.

\subsection{Less expensive for students and teachers:}

E-Learning users do not need high-end configured computers to run e-Learning applications.

They can run applications from the Cloud through their devices (PCs, mobile phones, tablets) having a minimal configuration with the Internet connectivity. Since data is created and accessible in the cloud, the user does not need to spend money for software licenses, software technical support and large memory for storing data in local machines.

\subsection{Centralized data storage:}

If the client computer crashes, there is almost no data lost because all data are stored in the cloud. 


\subsection{Virtualization:}

Virtualization is the most important feature of this type of architecture. It is very easy to create a clone of a virtual machine so that the downtime of clouds should be greatly reduced.

\subsection{High availability:}

Due to the integration of massive storage and high-performance computing power, this system can provide a better quality of service. The cloud computing system can automatically detect node failure and exclude it, which does not affect the normal operation of the entire system.

\subsection{Easy Implementation and Update:}

Updating, can be performed in a short period. Therefore, we will pay more attention to learner's needs, instead of devoting time to implementation and maintenance of the infrastructure.

\section{Conclusion}

Through this research, we were able to set up a middleware architecture, for the improvement of the governance of an academic institution by using the ESB integration architecture in its various distributed campuses.

The goal is to provide a modern and scalable architecture that combines cloud computing with the ESB application integration architecture to be used in higher education.

We have shown that the benefits of integrating an e-learning system into the cloud can be putted forward for their flexibility and scalability of resources such as high availability, storage, and IT Access to the network. Thus, the cost is calculated according to the number of servers used depending to the number of students.

Finally, the proposed architecture offers interesting possibilities for researchers who wish to deepen and explore the integration of applications of an information system.

By way of perspective, we will improve this architecture by setting up an e-Learning platform at a national level, so to be accessible by the different higher institutions in Morocco.

\section{REFERENCES}

[1] Bernard Manouvrier and, Laurent Ménard , "Application Integration: EAI, B2B, BPM and SOA",ISBN: 9781-84821-088-2, chapter 5, Published Online: 27 JAN 2010.

[2] BumSuk Jang and Young-guk Ha, "Improvement of Schema-Informed XML Binary Encoding Using Schema Optimization Method",Computers, Networks, Systems, and Industrial Applications, Springer, 2011.

[3] Chappel, Dave, “Enterprise Service Bus”, O'Reilly,2004.

[4] Christian Baun, Marcel Kunze, Jens Nimis, and Stefan Tai, "Cloud Computing Web-Based Dynamic IT Services," in Cloud Computing.: Springer Berlin Heidelberg, 2011, pp. 15--48.

[5] Dimitrios Zissis and Dimitrios Lekkas, "Addressingcloud computing security issues", Future GeberationComputer Systems, pp. 583-592, 2012. 
Abdellah Lalaoui Hassani, Khalifa Mansouri. Towards a Model of a Scalable Middleware Architecture Based on Cloud Computing, Application Reliable Integration and E-Learning Platforms in Academic Information Systems . Transactions on Machine Learning and Artificial Intelligence, Vol 5 No 4 August (2017); p: 651-664

[6] Faouzia MESSAOUDI, “DISPOSITIFS DE E-LEARNING : QUELS USAGES POUR AMELIORER LA FORMATION AU MAROC ? Du premier baromètre national du e-Learning à l'étude de cas de la formation continue", pp.58-95, 2013.

[7] Fenouillet, F \& Déro, M, “ Le e-learning est-il efficace ? Une analyse de la littérature anglo-saxonne, in Savoirs", vol. 12, n³, pp. 88-101,2006.

[8] FLETCHER J. D, “ Effective-ness and cost of interactive videodisc instruction ", Machine Mediated Learning, pp. 361-385, 1991.

[9] Guillaume SUPERCZYNSKI, “Alfresco 3.4 Travail collaboratif et GED avec la plate-forme Share”, Editions ENI, 2011.

[10] isga.ma, "GESFO System", 2008. [Online]. Available: http://isga.ma/fr/espace-etudiant.

[11] J.A. Hijar Miranda,Daniel Vazquez Sanchez, Dario Emmanuel Vazquez Ceballos,Erika Hernandez Rubio, Amilcar Meneses Viveros, Elena Fabiola Ruiz Ledezma, “Middleware to integrate heterogeneous Learning Management Systems and initial results", International Journal of Advanced Computer Science and Applications (IJACSA), Vol. 5, No. 10, 2014.

[12] Marinela Mircea and Anca loana Andreescu, "Using Cloud Computin, in Higher Education: A Strategy to Improve Agility in the Current Financial Crisis, IBIMA publishing", Article ID 875547, 2011.

[13] Mell, P., \& Grance, T, "The NIST Definition of Cloud Computing", 2012.[Online].Available: http://nvlpubs.nist.gov/nistpubs/Legacy/SP/nistspecialpublication800-145.pdf.

[14] Sebastian Pätzold, Sabine Rathmayer, Stephan Graf , " Proposal for the design and implementation of a modern system architecture and integration infrastructure in context of e-learning and exchange of relevant data ", pp. 1-8, 2014.

[15] T Mather, S Kumaraswamy, S Latif,Cloud Security and Privacy, O’REILLY, September 2009

[16] Vivek Kale, “Enhancing Enterprise Intelligence: Leveraging ERP, CRM, SCM, PLM, BPM, and BI”, ISBN: $978-$ 1-4987-0597-4, CRC Press, 2016. 